\title{
SYMMETRIES AND EXACT SOLUTIONS OF A NONLINEAR PRICING OPTIONS EQUATION
}

\author{
M.M. DYSHAEV, V.E. FEDOROV
}

\begin{abstract}
We study the group structure of the Schönbucher-Wilmott equation with a free parameter, which models the pricing options. We find a five-dimensional group of equivalence transformations for this equation. By means of this group we find four-dimensional Lie algebras of the admitted operators of the equation in the cases of two cases of the free term and we find a three-dimensional Lie algebra for other nonequivalent specifications. For each algebra we find optimal systems of subalgebras and the corresponding invariant solutions or invariant submodels.
\end{abstract}

Keywords: nonlinear partial differential equation, nonlinear Black-Scholes equation, Schönbucher-Wilmott model, pricing options, group analysis, invariant solution.

Mathematics Subject Classification: 58J70, 76M60, 91G99, 35A30

\section{INTRODUCTION}

A traditional model in the theory of financial markets is the Black-Scholes model [1, 2] described by the inverse heat equation with variable coefficients. However, practical studies show that due to made assumptions, this model is far from being adequate for real processes going on the financial markets (see [3]-[6]). This is why during last decades, the researchers proceeded to more complicated models of the dynamics of the financial markets, for instance, they study the models with a stochastic volatility [7], the models taking into consideration the transaction costs [8] as well as other models [3, 4]. Nonlinear models become more and more popular among the researchers, in particular, nonlinear models of Black-Sholes type. For instance, the transaction-cost model in [9] is of the form

$$
w_{t}+\frac{1}{2} \sigma^{2} x^{2} w_{x x}\left(1+2 \rho x w_{x x}\right)=0,
$$

where $t$ is the time, $x$ is the price of a share, $w$ is the option price, $\sigma$ is the volatility of the share, $\rho$ are transaction costs.

Another type of the models are ones with a reduced form of the stochastic differential equation (reduced-form SDE models) having the form

$$
w_{t}+\frac{\sigma^{2} x^{2} w_{x x}}{2\left(1-b x w_{x x}\right)^{2}}=0,
$$

where $b$ is the liquidity parameter [10]- -13$]$.

M.M. Dyshaev, V.E. Fedorov, Symmetries And EXACT SOlutions of A NONLinear PRICING OPTIONS EQUATION.

(C) Dyshaev M.M., Fedorov V.E. 2017.

The work of the second author is supported by the Goverment of Russia (law no. 211, 16.03.2013), agreement no. 02.A03.21.0011 and by the Ministery of Education and Science of Russia, task no. 1.6462.2017/BCh).

Sumitted December 28, 2015. 
As it was shown in works [11, 14, 15], the model of the cost of the hedge stategy on a illiquid market with taking into consideration the influence of the large trader operations can be represented as

$$
w_{t}+\frac{\sigma^{2} x^{2} w_{x x}}{2\left(1-\rho x \lambda(x) w_{x x}\right)^{2}}=0 .
$$

In this case $\rho$ is a parameter determining the influence of the the large trader operations, while $\lambda(x)$ is chosen so that to obtain the required form of payment. The values $\rho$ and $\lambda(x)$ can be determined by the present option prices on the market.

One more model of the formation of the options price is the so-called equilibrium model with the reaction-function model. The examples of using this model are given in [15, 16. Let us consider the equation

$$
w_{t}+\frac{\sigma^{2} x^{2} w_{x x}}{2\left(1-\rho \frac{g^{\prime}\left(\rho w_{x}\right)}{g\left(\rho w_{x}\right)} x w_{x x}\right)^{2}}=0
$$

corresponding to the equilibrium model called also Schönbucher-Wilmott model [17]. This equation describes the formation of the option prices on a illiquid market with taking into consideration the sizes of the open trader positions. In this case $x$ is the price of a base asset, $\sigma$ is the volatility, $\rho \geqslant 0$ is a parameter characterizing the size of the trader position w.r.t. the total volume of the traded base asset. The models taking into consideration the influence of the transaction costs and of hedge deals of the large traders on the base asset can be applied to some assets sold on the exchanges of the developing countries.

In works [14, [17], 18], 19], in the considered model (2), the reaction function $\psi=\psi\left(F_{t}, \rho \Phi_{t}\right)$ depended on the fundamental price of a share $F_{t}$ and of the the demand normalized volume of the large traders $\rho \Phi_{t}$. In fact, the function $\psi$ is the equilibrium price. It ensures the equilibrium between the price of the base asset, the quantity of the position of the large traders on this asset and the fundamental price of the base asset $F_{t}$. In work [19] it is of the form $\psi(f, \alpha)=f e^{\alpha}$, in works [14], [17] it is taken as $\psi(f, \alpha)=f /(1-\alpha)$. While deriving equation (2), it was assumed in [18] that $\psi(f, \alpha)=f g(\alpha)$ for some increasing function $g=g(\alpha)$. The assumption on the increasing of the function $g(\alpha)$ involved in the function $\psi$ was first made in work [16] and it is in a good agreement with practical observations, when the prices grows under the increasing of the large traders positions.

The methods for studying the mentioned models are quite various; these are numerical methods, the methods of the theory of time series, the theory of the neural networks, etc. [20]-[23]. As usually, once we deal with the processes modeled by differential equations, it is important to have exact solutions of such equations. In the case of nonlinear differential equations one of the most effective methods allowing to find such solutions are ones of the group analysis [24], [25]. The first studies of the group properties of the linear Black-Scholes equation were made in work [26] by N.Kh. Ibragimov and R.K. Gazizov. Apart from the linear equation, various nonlinear modifications of Black-Scholes equation are studied by the methods of the symmetry analysis. For instance, equation (1) was studied in this way in details in works by L.A. Bordag with co-authors [18], [27], 28]. Moreover, in works by L.A. Bordag and A. Mikaelyan [29], [30] there were obtained interesting results on symmetre and invariant solutions to equation (2).

This work is devoted to the group classification [24, 31] of equation (2) and to finding its exact solutions by the methods of the group analysis. In order to do it, in the second section we find equivalence transformations groups for this equation. By their means, in the third section we succeed to show that for the functions $v(\alpha)=g^{\prime}(\alpha) / g(\alpha)=\beta / \alpha$ corresponding to the power function $g(\alpha)=C \alpha^{\beta}$ and $v \equiv 1\left(g=C e^{\alpha}\right)$, the equation has a four-dimensional principal Lie algebra, while in the case not reduced to the mentioned by the equivalence transformations, the principal Lie algebra is three-dimensional. This result corrects the result of work [29], [30], in which three specific functions $v$ were found with additional symmetries; employing the obtained 
here equivalence transformations, it is easy to show the equivalence of two of them. The fourth, fifth and sixth section are devoted to finding invariant solutions and submodels of equations (2) with various Lie algebras (under different choices of the function $g$ ). In the seventh section we summarize the results of the study.

\section{Equivalence transformations of SchÖNbucher-Wilmott EQUATion}

We multiply equation (2) by the constant $\rho$, make the change $\rho w=u$ and redenote $g^{\prime}\left(\rho w_{x}\right) / g\left(\rho w_{x}\right)=v\left(u_{x}\right)$. We obtain the equation

$$
u_{t}+\frac{\sigma^{2} x^{2} u_{x x}}{2\left(1-x v\left(u_{x}\right) u_{x x}\right)^{2}}=0 .
$$

To find the functions $v=v\left(u_{x}\right)$, for which there arise symmetries additional to those defined by the core of the principal Lie algebra of equation (3), we need to find a continuous equivalence group of transformations for this equation. In order to do it, we write equation (3) as

$$
u_{t}+\frac{\sigma^{2} x^{2} u_{x x}}{2\left(1-x v u_{x x}\right)^{2}}=0,
$$

meaning that $v$ is an additional variable depending of the variables $t, x, u, u_{t}, u_{x}$. We seek the generators of the continuous equivalent transformations group as $Y=\tau \partial_{t}+\xi \partial_{x}+\eta \partial_{u}+\mu \partial_{v}$, where the functions $\tau, \xi, \eta$ depend on $t, x, u$, and $\mu$ depends on $t, x, u, u_{t}, u_{x}, v$. Hereinafter for the sake of shortness we use the notation $\frac{\partial}{\partial t} \equiv \partial_{t}$, etc. We complete equation (4) by the equations

$$
v_{t}=0, \quad v_{x}=0, \quad v_{u}=0, \quad v_{u_{t}}=0,
$$

meaning that in the original formulation of the problem, the function $v$ depends only on $u_{x}$.

We consider system (4), (5) as the manifold $\mathfrak{N}$ in the extended space of the corresponding variables. We apply by the extended operator

$$
\tilde{Y}=Y+\varphi^{t} \partial_{u_{t}}+\varphi^{x x} \partial_{u_{x x}}+\mu^{t} \partial_{v_{t}}+\mu^{x} \partial_{v_{x}}+\mu^{u} \partial_{v_{u}}+\mu^{u_{t}} \partial_{v_{u_{t}}}
$$

on the left hand side of system (4), (5), restrict the result of the action on the manifold $\mathfrak{N}$ and obtain the equation

$$
\begin{aligned}
& \varphi^{t}+\frac{\sigma^{2} x u_{x x} \xi}{\left(1-x v u_{x x}\right)^{3}}+\frac{\sigma^{2} x^{2}\left(1+x v u_{x x}\right) \varphi^{x x}}{2\left(1-x v u_{x x}\right)^{3}}+\left.\frac{\sigma^{2} x^{3} u_{x x}^{2} \mu}{\left(1-x v u_{x x}\right)^{3}}\right|_{\mathfrak{N}}=0, \\
& \left.\mu^{t}\right|_{\mathfrak{N}}=0,\left.\quad \mu^{x}\right|_{\mathfrak{N}}=0,\left.\quad \mu^{u}\right|_{\mathfrak{N}}=0,\left.\quad \mu^{u_{t}}\right|_{\mathfrak{N}}=0 .
\end{aligned}
$$

The coefficients of the operator $\tilde{Y}$ can be calculated by the continuation formulae, for instance,

$$
\mu^{t}=\widetilde{D}_{t}(\mu)-v_{t} \widetilde{D}_{t}(\tau)-v_{x} \widetilde{D}_{t}(\xi)-v_{u} \widetilde{D}_{t}(\eta)-v_{u_{t}} \widetilde{D}_{t}\left(\varphi^{t}\right)-v_{u_{x}} \widetilde{D}_{t}\left(\varphi^{x}\right)
$$

employing the differentiation operators

$$
\widetilde{D}_{t}=\partial_{t}+v_{t} \partial_{v}+v_{t t} \partial_{v_{t}}+v_{t x} \partial_{v_{x}}+v_{t u} \partial_{v_{u}}+v_{t u_{t}} \partial_{v_{u_{t}}}+v_{t u_{x}} \partial_{v_{u_{x}}}
$$

and so forth. Then equations (7) become

$$
\mu_{t}-v^{\prime}\left(u_{x}\right) \varphi_{t}^{x}=0, \quad \mu_{x}-v^{\prime}\left(u_{x}\right) \varphi_{x}^{x}=0, \quad \mu_{u}-v^{\prime}\left(u_{x}\right) \varphi_{u}^{x}=0, \quad \mu_{u_{t}}-v^{\prime}\left(u_{x}\right) \varphi_{u_{t}}^{x}=0 .
$$

Since

$$
\varphi^{x}=\eta_{x}+u_{x} \eta_{u}-u_{t} \tau_{x}-u_{t} u_{x} \tau_{u}-u_{x} \xi_{x}-u_{x}^{2} \xi_{u},
$$

we can write these equations as

$$
\begin{aligned}
& \mu_{t}-\left.v^{\prime}\left(u_{x}\right)\left(\eta_{t x}+u_{x} \eta_{t u}-u_{t} \tau_{t x}-u_{t} u_{x} \tau_{t u}-u_{x} \xi_{t x}-u_{x}^{2} \xi_{t u}\right)\right|_{\mathfrak{N}} \\
& \quad=\mu_{t}-v^{\prime}\left(u_{x}\right)\left(\eta_{t x}+u_{x} \eta_{t u}-u_{x} \xi_{t x}-u_{x}^{2} \xi_{t u}+\frac{\sigma^{2} x^{2} u_{x x} \tau_{t x}}{2\left(1-x v u_{x x}\right)^{2}}+\frac{\sigma^{2} x^{2} u_{x x} u_{x} \tau_{t u}}{2\left(1-x v u_{x x}\right)^{2}}\right)=0
\end{aligned}
$$




$$
\begin{aligned}
& \mu_{x}-\left.v^{\prime}\left(u_{x}\right)\left(\eta_{x x}+u_{x} \eta_{x u}-u_{t} \tau_{x x}-u_{t} u_{x} \tau_{x u}-u_{x} \xi_{x x}-u_{x}^{2} \xi_{x u}\right)\right|_{\mathfrak{N}} \\
& =\mu_{x}-v^{\prime}\left(u_{x}\right)\left(\eta_{x x}+u_{x} \eta_{x u}-u_{x} \xi_{x x}-u_{x}^{2} \xi_{x u}+\frac{\sigma^{2} x^{2} u_{x x} \tau_{x x}}{2\left(1-x v u_{x x}\right)^{2}}+\frac{\sigma^{2} x^{2} u_{x x} u_{x} \tau_{x u}}{2\left(1-x v u_{x x}\right)^{2}}\right)=0, \\
& \mu_{u}-\left.v^{\prime}\left(u_{x}\right)\left(\eta_{x u}+u_{x} \eta_{u u}-u_{t} \tau_{x u}-u_{t} u_{x} \tau_{u u}-u_{x} \xi_{x u}-u_{x}^{2} \xi_{u u}\right)\right|_{\mathfrak{N}} \\
& =\mu_{u}-v^{\prime}\left(u_{x}\right)\left(\eta_{x u}+u_{x} \eta_{u u}-u_{x} \xi_{x u}-u_{x}^{2} \xi_{u u}+\frac{\sigma^{2} x^{2} u_{x x} \tau_{x u}}{2\left(1-x v u_{x x}\right)^{2}}+\frac{\sigma^{2} x^{2} u_{x x} u_{x} \tau_{u u}}{2\left(1-x v u_{x x}\right)^{2}}\right)=0, \\
& \mu_{u_{t}}+v^{\prime}\left(u_{x}\right)\left(\tau_{x}+u_{x} \tau_{u}\right)=0 .
\end{aligned}
$$

Due to the identity

$$
\begin{aligned}
\varphi^{x x}= & \eta_{x x}+2 u_{x} \eta_{x u}+u_{x}^{2} \eta_{u u}+u_{x x} \eta_{u}-u_{t} \tau_{x x}-2 u_{t} u_{x} \tau_{x u}-2 u_{t x} \tau_{x}-u_{t} u_{x}^{2} \tau_{u u} \\
& -2 u_{x} u_{t x} \tau_{u}-u_{t} u_{x x} \tau_{u}-u_{x} \xi_{x x}-2 u_{x}^{2} \xi_{x u}-2 u_{x x} \xi_{x}-u_{x}{ }^{3} \xi_{u u}-3 u_{x} u_{x x} \xi_{u}
\end{aligned}
$$

equation (6) casts into the form

$$
\begin{aligned}
& \eta_{t}+u_{t} \eta_{u}-u_{t} \tau_{t}-u_{t}^{2} \tau_{u}-u_{x} \xi_{t}-u_{t} u_{x} \xi_{u}+\frac{\sigma^{2} x}{2\left(1-x v u_{x x}\right)^{3}}\left(2 u_{x x} \xi+2 x^{2} u_{x x}^{2} \mu\right. \\
& +x\left(1+x v u_{x x}\right)\left(\eta_{x x}+2 u_{x} \eta_{x u}+u_{x}^{2} \eta_{u u}+u_{x x} \eta_{u}-u_{t} \tau_{x x}-2 u_{t} u_{x} \tau_{x u}-2 u_{t x} \tau_{x}\right. \\
& \left.\left.-u_{t} u_{x}^{2} \tau_{u u}-2 u_{x} u_{t x} \tau_{u}-u_{t} u_{x x} \tau_{u}-u_{x} \xi_{x x}-2 u_{x}^{2} \xi_{x u}-2 u_{x x} \xi_{x}-u_{x}{ }^{3} \xi_{u u}-3 u_{x} u_{x x} \xi_{u}\right)\right)\left.\right|_{\mathfrak{N}} \\
& =\eta_{t}-\frac{\sigma^{2} x^{2} u_{x x} \eta_{u}}{2\left(1-x v u_{x x}\right)^{2}}+\frac{\sigma^{2} x^{2} u_{x x} \tau_{t}}{2\left(1-x v u_{x x}\right)^{2}}-\frac{\sigma^{4} x^{4} u_{x x}^{2} \tau_{u}}{4\left(1-x v u_{x x}\right)^{4}}-u_{x} \xi_{t}+\frac{\sigma^{2} x^{2} u_{x} u_{x x} \xi_{u}}{2\left(1-x v u_{x x}\right)^{2}} \\
& +\frac{\sigma^{2} x}{2\left(1-x v u_{x x}\right)^{3}}\left(2 u_{x x} \xi+2 x^{2} u_{x x}^{2} \mu+x\left(1+x v u_{x x}\right)\left(\eta_{x x}+2 u_{x} \eta_{x u}+u_{x}^{2} \eta_{u u}+u_{x x} \eta_{u}\right.\right. \\
& +\frac{\sigma^{2} x^{2} u_{x x} \tau_{x x}}{2\left(1-x v u_{x x}\right)^{2}}+\frac{\sigma^{2} x^{2} u_{x} u_{x x} \tau_{x u}}{\left(1-x v u_{x x}\right)^{2}}-2 u_{t x} \tau_{x}+\frac{\sigma^{2} x^{2} u_{x}^{2} u_{x x} \tau_{u u}}{2\left(1-x v u_{x x}\right)^{2}}-2 u_{x} u_{t x} \tau_{u} \\
& \left.\left.+\frac{\sigma^{2} x^{2} u_{x x}^{2} \tau_{u}}{2\left(1-x v u_{x x}\right)^{2}}-u_{x} \xi_{x x}-2 u_{x}^{2} \xi_{x u}-2 u_{x x} \xi_{x}-u_{x}{ }^{3} \xi_{u u}-3 u_{x} u_{x x} \xi_{u}\right)\right)=0 .
\end{aligned}
$$

Differentiating the latter equation w.r.t. $u_{t x}$, we obtain

$$
\left(1+x v u_{x x}\right)\left(\tau_{x}+u_{x} \tau_{u}\right)=0
$$

which implies $\tau=\tau(t)$. Therefore, equations (9)-(13) cast into the form

$$
\begin{aligned}
& \mu_{t}-v^{\prime}\left(u_{x}\right)\left(\eta_{t x}+u_{x} \eta_{t u}-u_{x} \xi_{t x}-u_{x}^{2} \xi_{t u}\right)=0 \\
& \mu_{x}-v^{\prime}\left(u_{x}\right)\left(\eta_{x x}+u_{x} \eta_{x u}-u_{x} \xi_{x x}-u_{x}^{2} \xi_{x u}\right)=0 \\
& \mu_{u}-v^{\prime}\left(u_{x}\right)\left(\eta_{x u}+u_{x} \eta_{u u}-u_{x} \xi_{x u}-u_{x}^{2} \xi_{u u}\right)=0 \\
& \mu_{u_{t}}=0 \\
& \eta_{t}-\frac{\sigma^{2} x^{2} u_{x x} \eta_{u}}{2\left(1-x v u_{x x}\right)^{2}}+\frac{\sigma^{2} x^{2} u_{x x} \tau^{\prime}(t)}{2\left(1-x v u_{x x}\right)^{2}}-u_{x} \xi_{t}+\frac{\sigma^{2} x^{2} u_{x} u_{x x} \xi_{u}}{2\left(1-x v u_{x x}\right)^{2}} \\
& +\frac{\sigma^{2} x}{2\left(1-x v u_{x x}\right)^{3}}\left(2 u_{x x} \xi+2 x^{2} u_{x x}^{2} \mu+x\left(1+x v u_{x x}\right)\left(\eta_{x x}+2 u_{x} \eta_{x u}+u_{x}^{2} \eta_{u u}\right.\right. \\
& \left.\left.+u_{x x} \eta_{u}-u_{x} \xi_{x x}-2 u_{x}^{2} \xi_{x u}-2 u_{x x} \xi_{x}-u_{x}{ }^{3} \xi_{u u}-3 u_{x} u_{x x} \xi_{u}\right)\right)=0 .
\end{aligned}
$$


We multiply equation (18) by $2\left(1-x v u_{x x}\right)^{3}$ to obtain

$$
\begin{aligned}
& 2\left(1-x v u_{x x}\right)^{3} \eta_{t}+\left(1-x v u_{x x}\right) \sigma^{2} x^{2} u_{x x}\left(\tau^{\prime}(t)-\eta_{u}\right)-2\left(1-x v u_{x x}\right)^{3} u_{x} \xi_{t} \\
& +\left(1-x v u_{x x}\right) \sigma^{2} x^{2} u_{x} u_{x x} \xi_{u}+\sigma^{2} x\left(2 u_{x x} \xi+2 x^{2} u_{x x}^{2} \mu+x\left(1+x v u_{x x}\right)\left(\eta_{x x}+2 u_{x} \eta_{x u}\right.\right. \\
& \left.\left.+u_{x}^{2} \eta_{u u}+u_{x x} \eta_{u}-u_{x} \xi_{x x}-2 u_{x}^{2} \xi_{x u}-2 u_{x x} \xi_{x}-u_{x}^{3} \xi_{u u}-3 u_{x} u_{x x} \xi_{u}\right)\right)=0 .
\end{aligned}
$$

Splitting equation (19) w.r.t. the variable $u_{x x}$, we get the coefficient $\eta_{t}-u_{x} \xi_{t}$ at $u_{x x}^{3}$ under the conditition $v \neq 0$ and this is why $\xi=\xi(x, u), \eta=\eta(x, u)$. Then by (14) we obtain that $\mu_{t}=0$ and equation (19) becomes

$$
\begin{aligned}
\left(1-x v u_{x x}\right) x u_{x x} & \left(\tau^{\prime}(t)-\eta_{u}\right)+\left(1-x v u_{x x}\right) x u_{x} u_{x x} \xi_{u}+2 u_{x x} \xi+2 x^{2} u_{x x}^{2} \mu \\
& +x\left(1+x v u_{x x}\right)\left(\eta_{x x}+2 u_{x} \eta_{x u}+u_{x}^{2} \eta_{u u}+u_{x x} \eta_{u}-u_{x} \xi_{x x}\right. \\
& \left.-2 u_{x}^{2} \xi_{x u}-2 u_{x x} \xi_{x}-u_{x}^{3} \xi_{u u}-3 u_{x} u_{x x} \xi_{u}\right)=0 .
\end{aligned}
$$

We differentiate this equation w.r.t. $t$ and get

$$
\tau^{\prime \prime}(t)=0, \quad \tau(t)=A t+B,
$$

and the equation casts into the form

$$
\begin{aligned}
\left(1-x v u_{x x}\right) x u_{x x}\left(A-\eta_{u}\right) & +\left(1-x v u_{x x}\right) x u_{x} u_{x x} \xi_{u}+2 u_{x x} \xi+2 x^{2} u_{x x}^{2} \mu \\
& +x\left(1+x v u_{x x}\right)\left(\eta_{x x}+2 u_{x} \eta_{x u}+u_{x}^{2} \eta_{u u}+u_{x x} \eta_{u}-u_{x} \xi_{x x}\right. \\
& \left.-2 u_{x}^{2} \xi_{x u}-2 u_{x x} \xi_{x}-u_{x}{ }^{3} \xi_{u u}-3 u_{x} u_{x x} \xi_{u}\right)=0 .
\end{aligned}
$$

Splitting (20) w.r.t. $u_{x x}$, we find the coefficient at $u_{x x}^{0}$ :

$$
x\left(\eta_{x x}+2 u_{x} \eta_{x u}+u_{x}^{2} \eta_{u u}-u_{x} \xi_{x x}-2 u_{x}^{2} \xi_{x u}-u_{x}^{3} \xi_{u u}\right)=0 .
$$

By splitting this equation w.r.t. $u_{x}$ we obtain the system

$$
\xi_{u u}=\eta_{x x}=0, \quad 2 \eta_{x u}=\xi_{x x}, \quad \eta_{u u}=2 \xi_{x u} .
$$

It follows from (21) that $\xi=C(x) u+D(x), \eta=E(u) x+F(u)$. Substituting these expression into (22), we obtain $2 E^{\prime}(u)=C^{\prime \prime}(x) u+D^{\prime \prime}(x)$. Then

$$
\begin{aligned}
& C^{\prime \prime \prime}(x)=D^{\prime \prime \prime}(x)=0, \quad C(x)=G x^{2}+H x+I, \quad D(x)=J x^{2}+K x+L, \\
& E^{\prime}(u)=G u+J, \quad E(u)=\frac{1}{2} G u^{2}+J u+M .
\end{aligned}
$$

Thus,

$$
\xi=G x^{2} u+H x u+I u+J x^{2}+K x+L, \quad \eta=\frac{1}{2} G x u^{2}+J x u+M x+F(u) .
$$

Substituting these expression into (23), we obtain the equation $F^{\prime \prime}(u)=3 G x+2 H$, which implies

$G=0, \quad F(u)=H u^{2}+N u+P, \quad \xi=H x u+I u+J x^{2}+K x+L, \quad \eta=J x u+M x+H u^{2}+N u+P$.

Equating to zero the coefficients at $u_{x x}$ and $u_{x x}^{2}$ in the left hand side of equation (20), we obtain the equations

$$
\begin{aligned}
& \mu=\left[\frac{A}{2}-\eta_{u}+2 u_{x} \xi_{u}+\xi_{x}\right] v \\
& A x+2 \xi-2 x \xi_{x}-2 x u_{x} \xi_{u}-x^{2} u_{x} \xi_{x x} v-2 x^{2} u_{x}^{2} \xi_{x u} v+2 x^{2} u_{x} \eta_{x u} v+x^{2} u_{x}^{2} \eta_{u u} v \\
& =A x+2 I u+2 L-2 J x^{2}-2 H x^{2} u_{x}-2 I x u_{x}=0 .
\end{aligned}
$$

The latter identity implies that $A=H=I=J=L=0$. Then

$$
\tau=B, \quad \xi=K x, \quad \eta=M x+N u+P, \quad \mu=(K-N) v .
$$


At that, identities (14)-(17) hold true as well.

Thus, the solutions to the system of equations determining the generators of the continuous equivalence transformations groups are given by (24). This leads us to the following statement.

Theorem 1. The basis of the Lie algebra of the infinitesimal operators of the equivalence transformations groups for equation (3) with a function $v$ not being identically zero is formed by the operators

$$
Y_{1}=\partial_{t}, \quad Y_{2}=\partial_{u}, \quad Y_{3}=x \partial_{u}, \quad Y_{4}=x \partial_{x}+u \partial_{u}, \quad Y_{5}=u \partial_{u}-v \partial_{v} .
$$

Here the element of the basis corresponding to the solution at $K=1, B=M=N=P=0$ is replaced by its sum with its element corresponding to the solution $N=1, B=K=M=P=0$ in order to minimizes the amount of basis operators involving the additional variable $v$.

In view of formula (8) we obtain the extensions of the basis operators

$$
\tilde{Y}_{1}=\partial_{t}, \quad \tilde{Y}_{2}=\partial_{u}, \quad \tilde{Y}_{3}=x \partial_{u}+\partial_{u_{x}}, \quad \widetilde{Y}_{4}=x \partial_{x}+u \partial_{u}, \quad \tilde{Y}_{5}=u \partial_{u}-v \partial_{v}+u_{x} \partial_{u_{x}} .
$$

This implies that the basis of the core of principal Lie algebra for equation (3) is formed by the operators $Y_{1}, Y_{2}, Y_{4}$, whose extensions do not involve the additional variables $v, u_{x}$.

Corollary 1. The basis of the cores of principal Lie algebras of equation (3) with a function $v$ not being identically zero is formed by the operators $X_{1}=\partial_{t}, X_{2}=\partial_{u}, X_{3}=x \partial_{x}+u \partial_{u}$.

\section{Group Classification of EQUATion}

We consider the Lie algebra obtained by the projections of operators (25) on the subspace of the variables $v, u_{x}$, that is, the algebra with the basis

$$
Z_{1}=\partial_{u_{x}}, \quad Z_{2}=v \partial_{v}-u_{x} \partial_{u_{x}}
$$

First of all we observe that the operator $Y_{3}$ corresponds to the operator $Z_{1}$ and the operator $-Y_{5}$ corresponds to the operator $Y_{3}$.

Non-zero structural constants for this Lie algebra are $c_{12}^{1}=-1, c_{21}^{1}=1$. By the formula $E_{\alpha}=$ $c_{\alpha \beta}^{\gamma} e^{\beta} \partial_{e^{\gamma}}$ we find the generators of the inner automorphisms of the Lie algebra $E_{1}=-e^{2} \partial_{e^{1}}$, $E_{2}=e^{1} \partial_{e^{1}}$ and the associated transformations groups:

$$
E_{1}: \bar{e}^{1}=e^{1}-e^{2} a_{1}, \quad E_{2}: \bar{e}^{1}=e^{1} e^{a_{2}} .
$$

Here $e^{i}$ is the coefficient at the operator $Z_{i}$ in the expansions of an element of the considered Lie algebra in its basis.

Let $e^{2} \neq 0$, then $e^{1}=0$ by $E_{1}$. We obtain the subalgebra with the basis $Z_{2}$. Otherwise we have a one-dimensional algebra with the basis $Z_{1}$. Thus, the optimal system of one-dimensional subalgebras of the algebra $L_{2}$ with basis (26) is of the form $\Theta_{1}=\left\{\left\langle Z_{1}\right\rangle,\left\langle Z_{2}\right\rangle\right\}$.

We calculate the expressions $\left.Z\left(V\left(u_{x}\right)-v\right)\right|_{v=V}=0$ for the operators $Z$ in the optimal system:

$$
\begin{aligned}
& \left.Z_{1}\left(V\left(u_{x}\right)-v\right)\right|_{v=V}=V^{\prime}=0, \quad V \equiv \beta ; \\
& \left.Z_{2}\left(V\left(u_{x}\right)-v\right)\right|_{v=V}=-V-u_{x} V^{\prime}=0, \quad V=\frac{\beta}{u_{x}} .
\end{aligned}
$$

The first case by the equivalence transformation $Y_{5}$ is reduced to the case $V \equiv 1$. In the second case the equivalence transformations found in the previous section can not change the constant $\beta$.

For each basis operator in the optimal system we calculate the projection of the corresponding generator of the equivalence transformations group $\left(Y_{3}\right.$ or $\left.-Y_{5}\right)$ on the subspace of the variables $t, x, u$. Up to a multiplicative constant, we obtain the correspondence $Z_{1}: x \partial_{u}, Z_{2}: u \partial_{u}$. This is why in the case $v \equiv 1$ we have the additional symmetry $x \partial_{u}$, while in the cases

$$
v=\beta u_{x}^{-1}, \quad \beta \in \mathbb{R},
$$

the additional symmetry is $u \partial_{u}$. 
Theorem 2. 1. The basis of the principal Lie algebra of the equation

$$
u_{t}+\frac{\sigma^{2} x^{2} u_{x x}}{2\left(1-x u_{x x}\right)^{2}}=0
$$

is of the form $X_{1}=\partial_{t}, X_{2}=\partial_{u}, X_{3}=x \partial_{x}+u \partial_{u}, X_{4}=x \partial_{u}$.

2. The basis of the principal Lie algebra of the equations

$$
u_{t}+\frac{\sigma^{2} x^{2} u_{x x}}{2\left(1-\frac{\beta x u_{x x}}{u_{x}}\right)^{2}}=0, \quad \beta \in \mathbb{R},
$$

is of the form $X_{1}=\partial_{t}, X_{2}=\partial_{u}, X_{3}=x \partial_{x}, X_{4}=u \partial_{u}$.

3. If the function $v$ is not reduced to the above cases by the equivalence transformation, the principal Lie algebra of the equation

$$
u_{t}+\frac{\sigma^{2} x^{2} u_{x x}}{2\left(1-x v\left(u_{x}\right) u_{x x}\right)^{2}}=0
$$

coincide with the core of the principal Lie algebra and is the form $X_{1}=\partial_{t}, X_{2}=\partial_{u}, X_{3}=$ $x \partial_{x}+u \partial_{u}$.

In the second statement of the theorem we have employed a linear transformation of the basis to simplify its elements.

Remark 1. In work [29], apart from the choices of the function $v$ in Statement 2 and 3 in Theorem 2, extra two cases were indicated:

$$
v=\beta\left(1-u_{x}\right)^{-1}, \quad \beta \in \mathbb{R},
$$

see [29, Sect. 4, Thm. 4.2.1, Stm. 4], having additional fourth symmetry. However, by means of the transformation $v \rightarrow-v$, which is obviously the inner automorphism of the corresponding equivalence transformations group of the algebra $L_{5}$ in Theorem 1 , and also employing the equivalence transformation generated by the operator $Y_{3}$, which shifts the argument of the function $v$, we can transform these cases to form (27) in Statement 2 of Theorem 2. The described transformations correspond to the change of variables $\bar{t}=t, \bar{x}=x, \bar{u}=x-u$, where the line over a symbol denotes a new variable. It is easy to check that under such transformations the Lie algebra corresponding to the case (28) is reduced to that of the equation in case (27). This is why from the point of view of the group analysis, cases (27) and (28) are equivalent.

\section{INVARIANT SUBMODELS IN THE GENERAL CASE}

We consider the equation

$$
u_{t}+\frac{\sigma^{2} x^{2} u_{x x}}{2\left(1-x v\left(u_{x}\right) u_{x x}\right)^{2}}=0,
$$

whose Lie algebra $L_{3}$ has the basis

$$
X_{1}=\partial_{t}, \quad X_{2}=\partial_{u}, \quad X_{3}=x \partial_{x}+u \partial_{u} .
$$

Its nonzero structural constants are $c_{23}^{2}=1, c_{32}^{2}=-1$, and hence, the groups of the inner automorphisms are of the form $E_{2}: \bar{e}^{2}=e^{2}+a_{1} e^{3}, E_{3}: \bar{e}^{2}=e^{2} e^{-a_{2}}$. Employing them, let us seek an optimal system of one-dimensional subalgebras of this Lie algebra $L_{3}$. The infinitesimal generators of the required basis operators for these subalgebras are of the form $X=\sum_{k=1}^{3} e^{k} X_{k}=\left(e^{1}, e^{2}, e^{3}\right)$.

1. Let $e^{3} \neq 0$, then by means of $E_{2}$ we obtain $e^{2}=0$, and hence, the basis vector of the subalgebra is of the form $X=(a, 0,1), a \in \mathbb{R}$. 
2.1. As $e^{3}=0, e^{1} \neq 0, e^{2} \neq 0$, we obtain $X=(1,1,0)$. At that, we used the inner automorphisms $E_{3}$ and $\bar{e}^{2}=-e^{2}$.

2.2. The cases $X=(1,0,0)$ and $X=(0,1,0)$ remain.

Lemma 1. The optimal system of one-dimensional subalgebras of Lie algebra $L_{3}$ with basis (30) is of the form

$$
\Theta_{1}=\left\{\left\langle X_{1}\right\rangle,\left\langle X_{2}\right\rangle,\left\langle X_{1}+X_{2}\right\rangle,\left\langle a X_{1}+X_{3}\right\rangle, a \in \mathbb{R}\right\} .
$$

Employing the operators in the optimal system, let us find invariant submodels of equation (29) and, if it is possible, its invariant solutions. The results of the study is presented in Table 1 , where in the second column we write an one-dimensional subalgebra in the optimal system, third column contains its invariants, while in the fourth column we provide the corresponding invariant submodel of the original equations or its solution under the assumption that the restrictions implied by the equation or the domain of the function $v$ are satisfied. The symbols $A, B$ stand for arbitrary integration constants.

Table 1.

\begin{tabular}{|l|l|l|l|}
\hline & Subalgebra & Invariants & Submodel or solution \\
\hline 1 & $\left\langle X_{1}\right\rangle$ & $x, u$ & $u=A x+B$ \\
\hline 2 & $\left\langle X_{2}\right\rangle$ & $t, x$ & no \\
\hline 3 & $\left\langle X_{1}+X_{2}\right\rangle$ & $u-t, x$ & $\begin{array}{l}\sigma^{2} x^{2} \psi^{\prime}=-2\left(1-x v(\psi) \psi^{\prime}\right)^{2} \neq 0, \\
u=t+\varphi(x), \varphi^{\prime}=\psi\end{array}$ \\
\hline 4 & $\left\langle X_{3}\right\rangle$ & $t, x^{-1} u$ & $u=A x$ \\
\hline 5 & $\left\langle a X_{1}+X_{3}\right\rangle$, & $\begin{array}{l}a \ln |x|-t, \\
x^{-1} u\end{array}$ & $\begin{array}{l}u=A x \text { or } 2\left(1-\left(a \varphi^{\prime}+a^{2} \varphi^{\prime \prime}\right) v\left(\varphi+a \varphi^{\prime}\right)\right)^{2}= \\
\sigma^{2}\left(a \varphi^{\prime}+a^{2} \varphi^{\prime \prime}\right) / \varphi^{\prime} \neq 0, u=x \varphi(a \ln |x|-t)\end{array}$ \\
\hline
\end{tabular}

5. INVARIANT SOLUTIONS AND SUBMODELS IN THE CASE $v \equiv \beta$

The equation

$$
u_{t}+\frac{\sigma^{2} x^{2} u_{x x}}{2\left(1-\beta x u_{x x}\right)^{2}}=0, \quad \beta \neq 0
$$

has the Lie algebra $L_{4}$ with the basis

$$
X_{1}=\partial_{t}, \quad X_{2}=\partial_{u}, \quad X_{3}=x \partial_{x}+u \partial_{u}, \quad X_{4}=x \partial_{u} .
$$

The nonzero structural constants of this algebra are $c_{23}^{2}=1, c_{32}^{2}=-1$, and hence, its groups of inner automorphisms are $E_{2}: \bar{e}^{2}=e^{2}+a_{1} e^{3}, E_{3}: \bar{e}^{2}=e^{2} e^{-a_{2}}$. Let us find the optimal system of one-dimensional subalgebras of this Lie algebra $L_{4}$.

1. Let $e^{3} \neq 0$, then by $E_{2}$ we obtain $e^{2}=0$ and hene, the basis vector of the subalgebra is of the form $X=(a, 0,1, b), a, b \in \mathbb{R}$.

2.1. As $e^{3}=0, e^{2} \neq 0$, we obtain the following.

2.1.1. If $e^{1} \neq 0$, then $X=(1,1,0, a), a \in \mathbb{R}$. At that, we employed the inner automorphisms $E_{3}$ and $\bar{e}^{2}=-e^{2}$.

2.1.2. Let $e^{1}=0$, then $X=(0,1,0,1)$ or $X=(0,1,0,0)$. In the first case we have also employed $E_{3}$ and $\bar{e}^{2}=-e^{2}$.

2.2. If $e^{2}=e^{3}=0$, then $X=(1,0,0, a), a \in \mathbb{R}$, or $X=(0,0,0,1)$.

Lemma 2. The optimal system of one-dimensional subalgebra of the algebra $L_{4}$ with basis (32) is of the form

$$
\Theta_{1}=\left\{\left\langle X_{2}\right\rangle,\left\langle X_{4}\right\rangle,\left\langle X_{2}+X_{4}\right\rangle,\left\langle X_{1}+a X_{4}\right\rangle,\left\langle X_{1}+X_{2}+a X_{4}\right\rangle,\left\langle a X_{1}+X_{3}+b X_{4}\right\rangle, a, b \in \mathbb{R}\right\} .
$$

For the one-dimensional subalgebras in the optimal system we obtain Table 2. 


\begin{tabular}{|c|c|c|c|}
\hline & Subalgebra & Invariants & Submodel, solution, restrictions \\
\hline 1 & $\left\langle X_{2}\right\rangle$ & $t, x$ & no \\
\hline 2 & $\left\langle X_{4}\right\rangle$ & $t, x$ & no \\
\hline 3 & $\left\langle X_{2}+X_{4}\right\rangle$ & $t, x$ & no \\
\hline 4 & $\left\langle X_{1}\right\rangle$ & $x, u$ & $u=A x+B$ \\
\hline 5 & $\begin{array}{l}\left\langle X_{1}+a X_{4}\right\rangle \\
a \neq 0\end{array}$ & $x, u-a t x$ & $\begin{array}{l}u=a t x+A_{a \beta \sigma} x \ln |x|+A x+B, \\
A_{a \beta \sigma}=\frac{2 \beta-\frac{\sigma^{2}}{2 a} \pm \sqrt{\frac{\sigma^{4}}{4 a^{2}}-\frac{2 \beta \sigma^{2}}{a}}}{2 \beta^{2}}, \sigma \neq 0, \\
\frac{\sigma^{4}}{4 a^{2}}-\frac{2 \beta \sigma^{2}}{a} \geqslant 0\end{array}$ \\
\hline 6 & $\left\langle X_{1}+X_{2}+a X_{4}\right\rangle$ & $\begin{array}{l}x, \\
u-(1+a x) t\end{array}$ & $\begin{array}{l}\varphi^{\prime \prime}=\frac{2 \beta-\frac{\sigma^{2} x}{2(1+a x)} \pm \sqrt{\frac{\sigma^{4} x^{2}}{4(1+a x)^{2}}-\frac{2 \beta \sigma^{2} x}{1+a x}}}{2 \beta^{2} x} \\
\sigma \neq 0, u=(1+a x) t+\varphi(x)\end{array}$ \\
\hline 7 & $\begin{array}{l}\left\langle X_{3}+b X_{4}\right\rangle \\
b \neq 1 / \beta\end{array}$ & $t, \frac{u}{x}-b \ln |x|$ & $u=b x \ln |x|+A x-\frac{b \sigma^{2} t x}{2(1-\beta b)^{2}}$ \\
\hline 8 & $\begin{array}{l}\left\langle a X_{1}+X_{3}+\right. \\
\left.b X_{4}\right\rangle, a \neq 0\end{array}$ & $\begin{array}{l}a \ln |x|-t, \\
\frac{u}{x}-b \ln |x|\end{array}$ & $\begin{array}{l}u=A x \text { as } b=0 \text { or } \\
\sigma^{2}\left(b+a \varphi^{\prime}+a^{2} \varphi^{\prime \prime}\right) / \varphi^{\prime}=2(1-\beta(b+ \\
\left.\left.a \varphi^{\prime}+a^{2} \varphi^{\prime \prime}\right)\right)^{2} \neq 0, \\
u=b x \ln |x|+x \varphi(a \ln |x|-t)\end{array}$ \\
\hline
\end{tabular}

Table 2 .

6. INVARIANT SOLUTIONS AND SUBMODELS IN THE CASE $v=\beta u_{x}^{-1}$

For the equation

$$
u_{t}+\frac{\sigma^{2} x^{2} u_{x x}}{2\left(1-\frac{\beta x u_{x x}}{u_{x}}\right)^{2}}=0, \quad \beta \neq 0,
$$

the basis of the Lie algebra $L_{4}$ is of the form

$$
X_{1}=\partial_{t}, \quad X_{2}=\partial_{u}, \quad X_{3}=x \partial_{x}, \quad X_{4}=u \partial_{u} .
$$

The nonzero structural constants are $c_{24}^{2}=1, c_{42}^{2}=-1$, and this is why the structure of this Lie algebra does not differ from the algebra in the previous section up to a renumeration of yhe operators $X_{3}, X_{4}$. This observation and Lemma 2 yield immediately the following statement.

Lemma 3. The optimal system of one-dimensional algebras of the algebra $L_{4}$ with basis (34) is of the form

$$
\Theta_{1}=\left\{\left\langle X_{2}\right\rangle,\left\langle X_{3}\right\rangle,\left\langle X_{2}+X_{3}\right\rangle,\left\langle X_{1}+a X_{3}\right\rangle,\left\langle X_{1}+X_{2}+a X_{3}\right\rangle,\left\langle a X_{1}+b X_{3}+X_{4}\right\rangle, a, b \in \mathbb{R}\right\} .
$$

Employing the operators in the optimal system, we find the invariant submodels of equation (33) and, if it is possible, its invariant solutions (Table 3 ).

As $\beta=-1$, the operator $X_{2}+X_{3}$ has no invariant solutions.

As $a \neq 0$, the invariant submodel for the operators $X_{1}+a X_{3}$ is of the form

$$
\sigma^{2} \varphi^{\prime}\left(\varphi^{\prime \prime}-\varphi^{\prime}\right)=2 a\left(\varphi^{\prime}-\beta\left(\varphi^{\prime \prime}-\varphi^{\prime}\right)\right)^{2} \neq 0 .
$$

We denote the argument of the function $\varphi$ by $z$. By the substitution $\zeta=e^{z}$ and by decreasing the order of the equation we obtain $\sigma^{2} \zeta \psi \psi^{\prime}=2 a\left(\psi-\beta \zeta \psi^{\prime}\right)^{2}$, where $\psi(\zeta)=\frac{d}{d \zeta} \varphi(\ln \zeta)$. Making the inverse change $\xi(z)=\psi\left(e^{z}\right)$, we obtain the quadratic equation for the derivative

$$
\sigma^{2} \xi \xi^{\prime}=2 a\left(\xi-\beta \xi^{\prime}\right)^{2} .
$$

As $a \geqslant-\frac{\sigma^{2}}{8 \beta}$, in the case of positive $\beta$, and as $a \leqslant-\frac{\sigma^{2}}{8 \beta}$ for negative $\beta$, by the integration we obtain $\xi(z)=A e^{C_{1} z}$, where

$$
C_{1}=\frac{4 a \beta+\sigma^{2} \pm \sigma \sqrt{\sigma^{2}+8 a \beta}}{4 a \beta^{2}}
$$


Therefore, the invariant solution is of the form shown in the fifth row of the table.

The invariant solution of the subalgebra $\left\langle b X_{3}+X_{4}\right\rangle, b \neq 0$, (row 9 in the table) is in fact the solution with an arbitrary nonzero power of $|x|$ (to avoid $u_{x}=0$ ) and the corresponding to this power coefficient at $t$ in the argument of the exponential function in the same term of the solution. Its particular case is the invariant solution for $\left\langle X_{1}+a X_{3}\right\rangle, a \neq 0$ (row 5).

Table 3.

\begin{tabular}{|c|c|c|c|}
\hline & Subalgebra & Invariants & Submodel, solution, restrictions \\
\hline 1 & $\left\langle X_{2}\right\rangle$ & $t, x$ & no \\
\hline 2 & $\left\langle X_{3}\right\rangle$ & $t, u$ & no \\
\hline 3 & $\left\langle X_{2}+X_{3}\right\rangle$ & $t, u-\ln |x|$ & $u=\ln |x|+\frac{\sigma^{2} t}{2(1+\beta)^{2}}+A, \beta \neq-1$ \\
\hline 4 & $\left\langle X_{1}\right\rangle$ & $x, u$ & $u=A x+B, A \neq 0$ \\
\hline 5 & $\begin{array}{l}\left\langle X_{1}+a X_{3}\right\rangle \\
a \neq 0\end{array}$ & $\ln |x|-a t, u$ & $\begin{array}{l}u=A e^{-a C t}|x|^{C}+B, A \neq 0, \\
C=\frac{4 a \beta(\beta+1)+\sigma^{2} \pm \sigma \sqrt{\sigma^{2}+8 a \beta}}{4 a \beta^{2}} \neq 0, \\
\sigma \neq 0, \sigma^{2}+8 a \beta \geqslant 0\end{array}$ \\
\hline 6 & $\left\langle X_{1}+X_{2}+a X_{3}\right\rangle$ & $\begin{array}{l}\ln |x|-a t \\
u-t\end{array}$ & $\begin{array}{l}2\left(a \varphi^{\prime}-1\right)\left(\varphi^{\prime}-\beta\left(\varphi^{\prime \prime}-\varphi^{\prime}\right)\right)^{2}= \\
\sigma^{2} \varphi^{\prime 2}\left(\varphi^{\prime \prime}-\varphi^{\prime}\right), \varphi(z) \neq A e^{(1+1 / \beta) z}+B \\
u=t+\varphi(\ln |x|-a t)\end{array}$ \\
\hline 7 & $\left\langle X_{4}\right\rangle$ & $t, x$ & no \\
\hline 8 & $\begin{array}{l}\left\langle a X_{1}+X_{4}\right\rangle \\
a \neq 0\end{array}$ & $x, e^{-t / a} u$ & $\begin{array}{l}a \sigma^{2} x^{2} \varphi^{\prime 2} \varphi^{\prime \prime}=2 \varphi\left(\varphi^{\prime}-\beta x \varphi^{\prime \prime}\right)^{2} \neq 0 \\
u=e^{t / a} \varphi(x)\end{array}$ \\
\hline 9 & $\begin{array}{l}\left\langle b X_{3}+X_{4}\right\rangle, \\
b \neq 0\end{array}$ & $t,|x|^{-1 / b} u$ & $\begin{array}{l}u=A e^{\frac{\sigma^{2}(b-1) t}{2(b(\beta+1)-\beta)^{2}}}|x|^{1 / b}+B \\
A \neq 0, b \neq \frac{\beta}{\beta+1} \quad \beta \neq-1\end{array}$ \\
\hline 10 & $\begin{array}{l}\left\langle a X_{1}+b X_{3}+X_{4}\right\rangle \\
a \neq 0, b \neq 0\end{array}$ & $\begin{array}{l}a \ln |x|-b t \\
e^{-t / a} u\end{array}$ & $\begin{array}{l}a^{2} \sigma^{2} \varphi^{\prime 2}\left(a \varphi^{\prime \prime}-\varphi^{\prime}\right)= \\
2\left(a b \varphi^{\prime}-\varphi\right)\left(\varphi^{\prime}-\beta\left(a \varphi^{\prime \prime}-\varphi^{\prime}\right)\right)^{2} \\
\varphi(z) \neq A e^{\frac{1}{a}(1+1 / \beta) z}+B \\
u=e^{t / a} \varphi(a \ln |x|-b t)\end{array}$ \\
\hline
\end{tabular}

\section{Exact solutions to Schönbucher-Wilmott EQUATION EQUATion}

Let us analyse the obtained results in terms of the original problem. The functions $v \equiv \beta$ correspond to the model

$$
w_{t}+\frac{\sigma^{2} x^{2} w_{x x}}{2\left(1-\beta \rho x w_{x x}\right)^{2}}=0
$$

with the function $g(\alpha)=G e^{\beta \alpha}$ at the integration constant $G$. We restrict ourselves by considering the found exact solutions. Returning back from $u$ to the function $w$, in view of the arbitrary choice of some constants, we obtain the solutions

$w(t, x)=A x+B$

$w(t, x)=\frac{a t x}{\rho}+\frac{2 \beta-\frac{\sigma^{2}}{2 a} \pm \sqrt{\frac{\sigma^{4}}{4 a^{2}}-\frac{2 \beta \sigma^{2}}{a}}}{2 \beta^{2} \rho} x \ln |x|+B x+C, \quad a \neq 0, \quad \sigma \neq 0, \quad \frac{\sigma^{4}}{4 a^{2}}-\frac{2 \beta \sigma^{2}}{a} \geqslant 0 ;$ $w(t, x)=\frac{b}{\rho} x \ln |x|-\frac{b \sigma^{2} t x}{2 \rho(1-\beta b)^{2}}+A x+B, \quad b \neq \frac{1}{\beta}$.

In the latter case the family of invariant solutions of the operators $X_{3}+b X_{4}, b \neq \frac{1}{\beta}$ is extended by the admitted groups of the shifts in the variable $u$ corresponding to the operator $X_{2}$.

By the equivalence transformations corresponding to the operator $Y_{3}$, the functions $v=\frac{\beta}{u_{x}}$ can be reduced to the equivalent form $v=\frac{\beta}{u_{x}+\gamma}, \gamma \in \mathbb{R}$. These functions correspond to the 
function $g(\alpha)=G(\alpha+\gamma)^{\beta}$ and the model

$$
w_{t}+\frac{\sigma^{2} x^{2} w_{x x}}{2\left(1-\frac{\beta x w_{x x}}{w_{x}+\frac{\gamma}{\rho}}\right)^{2}}=0 .
$$

It is easy to see that solutions (35) are related with the solutions of equation (33) by the identity $w(t, x)=(u(t, x)-\gamma x) \rho^{-1}$. This is why in accordance with the table in the previous section, equation (35) has exact solutions

$$
\begin{aligned}
& w(t, x)=A x+B, \quad A \neq-\frac{\gamma}{\rho} \\
& w(t, x)=A \ln |x|+\frac{A \sigma^{2} t}{2(1+\beta)^{2}}+B-\frac{\gamma}{\rho} x, \quad A \neq 0, \quad \beta \neq-1 \\
& w(t, x)=A e^{-\frac{\sigma^{2} C(C-1) t}{2(1-\beta(C-1))^{2}}} x^{C}+B-\frac{\gamma}{\rho} x, \quad A C \neq 0 .
\end{aligned}
$$

At that, the class of solutions $u(t, x)$ to equation (33) invariant w.r.t. the operator $X_{2}+X_{3}$, is extended by means of the action of the admitted operator $X_{4}$ and this is the reason of the presence of the factor $A$ in the first two terms.

We mention that in work [29] the symmetries of model (35) in the cases $\gamma=0$ and $\gamma \neq 0$ are studied independently. We can omit such study if we establish a passage from one symmetry to the other by means of the equivalence transformations group of the studied class of the equations, see Remark 1.

It is clear that we keep the condition on monotonous increasing of the function $g(\alpha)$, then in the considered cases of the function $g$ we need to impose the condition $\beta>0$.

\section{BIBLIOGRAPHY}

1. F. Black, M. Scholes. The pricing of options and corporate liabilities // J. Politic. Econ. 81:3, 637-659 (1973).

2. F. Black. The pricing of commodity contracts // J. Finan. Economics. 3:1-2, 167-179 (1976).

3. J. Cox, S. Ross, M. Rubinstein. Option pricing: a simplified approach // J. Finan. Economics. 7:3, 229-263 (1979).

4. J. Duan. The GARCH option pricing model // Math. Finance. 5:1, 13-32 (1995).

5. E. Derman, N. Taleb. The illusions of dynamic replication // Quantit. Finance. 5:4, 323-326 (2005).

6. E. G. Haug, N. N. Taleb. Option traders use (very) sophisticated heuristics, never the BlackScholes-Merton formula // J. Econom. Behavior Organiz. 77:2, 97-106 (2011).

7. J. C. Hull, A. White. The pricing of options on assets with stochastic volatilities // J. Finan. 42:2, 281-300 (1987).

8. H. E. Leland. Option pricing and replication with transactions costs // J. Finance. 40:5, 1283-1301 (1985).

9. U. Cetin, R. Jarrow, P. Protter. Liquidity risk and arbitrage pricing theory // Finance and Stochastic. 8:3, 311-341 (2004).

10. R. Frey. Market illiquidity as a source of model risk in dynamic hedging // in "Model Risk", ed. R. Gibson. Risk Publ., London, 125-136 (2000).

11. R. Frey, P. Patie. Risk Management for Derivatives in Illiquid Markets: a Simulation Study // in "Advances in Finance and Stochastics", eds. K. Sandmann and P. Schönbucher. Springer, Berlin (2002).

12. M. Jandaćka, D. Śevćović. On the risk-adjusted pricing-methodology-based valuation of vanilla options and explanation of the volatility smile // J. Appl. Math. 2005:3, 253-258 (2005).

13. H. Liu, J. Yong. Option pricing with an illiquid underlying asset market // J. Econom. Dynam Contr. 29:12, 2125-2156 (2005). 
14. R. Frey, A. Stremme. Market volatility and feedback effects from dynamic hedging // Math Finan. 7:4, 351-374 (1997).

15. R. Frey. Perfect option replication for a large trader // Finance and Stochastics. 2:2, 115-148 (1998).

16. R. A. Jarrow. Derivative securities markets, market manipulation and option pricing theory // J. Finan. Quantit. Anal. 29:2, 241-261 (1994).

17. P. Wilmott, P. Schonbucher. The feedback-effect of hedging in illiquid markets // SIAM J. Appl. Math. 61:1, 232-272 (2000).

18. L.A. Bordag, R. Frey. Pricing options in illiquid markets: symmetry reductions and exact solutions // in "Nonlinear Models in Mathematical Finance: Research Trends in Option Pricing", ed. M. Ehrhardt. Ch. 3. Nova Science Publishers, New York. 103-130 (2008).

19. E. Platen, M. Schweizer. On feedback effects from hedging derivatives // Math. Finan. 8:1, 67-84 (1998).

20. P. Brandimarte. Numerical methods in finance 83 economics. John Wiley \& Sons, New York (2004).

21. G. Bakshi, C. Cao, Z. Chen. Empirical performance of alternative option pricing models // J. Finan. 52:5, 2003-2049 (1997).

22. M. J. Morelli, G. Montagna, O. Nicrosini, M. Treccani, M. Farina, P. Amato. Pricing financial derivatives with neural networks // Physica A. 338:1-2, 160-165 (2004).

23. S. Kou. A jump diffusion model for option pricing // Management Sci. 48:8, 1086-1101 (2002).

24. L.V. Ovsyannikov. Group analysis of differential equations. Nauka, Moscow (1978). [Academic Press, New York (1982)]

25. N.Kh. Ibragimov. Transformation groups applied to mathematical physics. Nauka, Moscow (1983). [Math. Appl. Soviet Series, 3. D. Reidel Publishing Company, Dordrecht (1985).]

26. R.K. Gazizov, N.H. Ibragimov. Lie symmetry analysis of differential equations in finance // Nonl. Dynamics. 17:4, 387-407 (1998).

27. L.A. Bordag, A.Y. Chmakova. Explicit solutions for a nonlinear model of financial derivatives // Int. J. Theor. Appl. Finan. 10:1, 1-21 (2007).

28. L.A. Bordag. On option-valuation in illiquid markets: invariant solutions to a nonlinear model // in "Mathematical Control Theory and Finance", eds. A. Sarychev, A. Shiryaev, M. Guerra and M. R. Grossinho. Springer, Berlin, 71-94 (2008).

29. A. Mikaelyan. Analytical study of the Schönbucher-Wilmott model of the feedback effect in illiquid markets // Master's thesis in financial mathematics, Halmstad University, Halmstad (2009).

30. L.A. Bordag, A. Mikaelyan. Models of self-financing hedging strategies in illiquid markets: symmetry reductions and exact solutions // J. Lett. Math. Phys. 96:1-3, 191-207 (2011).

31. Yu.A. Chirkunov, S.V. Khabirov. Elements of symmetry analysis of differential equations in continuous media. Novosibirsk State Technical Univ., Novosibirsk (2012). (in Russian).

Mikhail Mikhailovich Dyshaev,

Chelyabinsk State University,

Br. Kashirinykh st. 129,

454001, Chelyabinsk, Russia

E-mail: Mikhail.Dyshaev@gmail.com

Vladimir Evegn'evich Fedorov,

Chelyabinsk State University,

Laboratory of quantum topology,

Br. Kashirinykh str. 129,

454001, Chelyabinks, Russia

South Ural State University

(National Research University),

Lenin av., 76,

454080, Chelyabinsk, Russia

E-mail: kar@csu.ru 